\title{
قياس أثر التكامل الاقتصادي لمجموعة السلع الغذائية (دراسة حالة بين السعودية والامارات والكويت)
}

\author{
محمد فلاح رفاعي مفلح نوار \\ باحث دكتوراه بمعهد البحوث والدراسات الأفريقية - جامعة أسوان
}

محمد عبد الحفيظ عحم

أستاذ الاقتصاد الزراعي وعميد كلية الزراعة - جامعة سوهاج

حسن موسي رضوان

مدرس الاقتصاد الزراعي بالمعهد العالي للتعاون والإرشاد الزراعي بأسيوط 


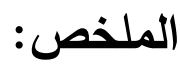

استهدف البحث بصفة رئيسية تحديد أثر قيام التكامل الاقتصادي بين السعودية

والامارات والكويت علي التجارة البينية فيما بينهم وعلي تجارتهم مع العالم الخارجي

لأهم والسلع الزراعية الغذائية ، ومن ثم في إمكانية التعاون والتبادل لتحقيق أفضل

طرق الاعتماد والاستفادة من التجارة الخارجية البينية بين السعودية وكل من الامارات

والكويت في ظل المتغيرات العالمية الحالية، واعتمد البحث في تحقيق أهدافه علي

استخدام أسلوب التحليل الكمي للتعرف علي اتجاهات وطبيعة التجارة البينية لأهم السلع الغذائية الرئيسية بين السعودية والامارات والكويت، كما اعتمد البحث للتعرف علي مدي إمكانية زيادة حجم التجارة الزراعية البينية من تلك السلع الغذائية علي استخدام نموذج تقدير أثر التكامل واعتمد البحث علي البيانات الثانوية المنشورة التي تصدرها المنظمة العربية للتنمية الزراعية.

الكلمات الإفتتاحية: التكامل الإقتصادى - السلع الغذائية - مصر والسعودية

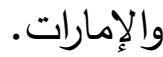

\section{Abstract}

Measuring the Impact of Economic Integration of the Food

Commodities Group

(A case study between Saudi Arabia, the UAE and Kuwait) 
The research aimed mainly to determine the impact of the establishment of economic integration between Saudi Arabia, the Emirates and Kuwait on the intra-trade between them and their trade with the outside world for the most important agricultural food commodities, and then on the possibility of cooperation and exchange to achieve the best methods of accreditation and benefit from the intra-foreign trade between Saudi Arabia and both the Emirates and Kuwait in The current global variables remained, and the research relied on achieving its goals on using a quantitative analysis method to identify the trends and nature of intra-regional trade for the most important major food commodities between Saudi Arabia, the Emirates and Kuwait. The impact of integration and the research was based on published secondary data issued by the Arab Organization for Agricultural Development.

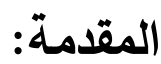

تشهذ المنطقة العربية حاليا الكثير من التغيرات السياسة والاقتصادية التي تؤكد أهمية دور التكامل الاقتصادي العربي في التتمية الثاملة بين الدول العربية حيث أثرت هذه التطورات السياسية الأخيرة في المنطقة تأثيرا سلبيا علي حجم الاستثارات بين الدول العربية مما أدي الي ارتفاع معدلات البطالة وضعف نتائج برامج العمل والتثغيل في معظم الدول العربية. 
جاء تأسيس مجلس التعاون الخليجي تجسيدا للمجموعة المقومات الاقتصادية الاجتماعية و الجغرافية و الثقافية و الحضارية المشتركة التي تربط بين الدول الأعضاء فيه، فقد تم في ع فبراير 1911 بالرياض التوقيع على وثيقة التأسيس من طرف وزراء الخارجية للدول الستة الأعضاء فيه (المملكة العببية السعودية، سلطنة عمان،البحرين، الإمارات،قر ، الكويت)، حيث حددت هذه الوثيقة السبل و المسارات التي يجب إتباعها من أجل تحقيق التكامل الاقتصادي بين الدول الأعضاء في مجلس التعاون الخليج

ففي المجال الاقتصادي تتشابه خصائص هذه الدول من حيث أن البترول والغار ومشتقاتهما )مباشرة أو غير مباشرة ( يشكلان العنصر الرئيسي لدكونات الدخل الوطني الإجمالي وللصادرات ـ كما تتثابه هياكل المالية العامة الي حد كبير مع وجود بعض الاختلافات المددوده بالأنظمة والإجراءات المالية ـ وتثثل دول مجلس التعاون حيزًا كبيرًا من الأرض يسكنه عدد قليل نسبيًا من البشر .إلا أن معدل النمو السكاني في جميع دول المجلس يعتبر من أعلى المعدلات في العالم .كما أن مستويات النمو الاقتصادي في الدول الست منقاربة.

و ما الانجازات التي حققها مجلس التعاون الخليجي في مسار التكامل الاقتصادي منذ تأسيسه، إلا ترجمة للإرادة السياسة القوية لأعضائه و طمح الكبير للثعوب دول الخليج العربية، فقد وصل التكامل الاقتصادي الخليجي إلى مرحلة جد 
متقدمة من مراحل التكامل الاقتصادي، والمتمثلة في السوق الخليجية المشتركة ، ولم يعد يفصله عن العملة الخليجية المشتركة إلا القليل، ليكتمل بذلك مشروع التكامل الاقتصادي بين دول مجلس التعاون الخليجي. إثكالية الدراسةة: ماهو واقع التكامل الاقتصادي العربي

وهذا ما يقتضي منا طرح جملة من التساؤلات التي تجسد إشكالية البحث والتي منها: 1- ماهي العوامل الدافعة الي التكامل الاقتصادي العربي. r- ماهي العوامل الدافعة الي تثكيل مجلس التعاون الخليج العربية. r- ماهي أبرز المحطات في مسيرة التكامل الدول الخليج العربية. ع - هل يمكن تعميم تجربة تكتل مجلس التعاون الخليجي علي الدول العربية.

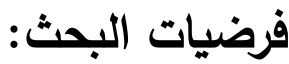

ا - التجارة العربية البينية تعزز من التكامل الاقتصادي العربي عن طريق خلق مشروعات اقتصادية متطورة .

r- من المشاكل التي تعيق التكامل الاقتصادي ضعف البنية الأساسية للتجارة العربية البينية. r- كلما ازداد التعاون بين دول الخليج العربية وثوقاً، سمح لها بتكوين مواقف موحدة، توافر لها قاعدة تفاوضية أفضل أمام الدول والتجمعات الاقتصادية الاخري. 


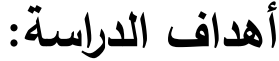

يهدف هذا البحث بصفة عامة تحديد أثر قيام التكامل الاقتصادي بين السعودية والامارات والكويت علي التجارة البينية فيما بينهم لأهم السلع الغذائية، ولتحقيق ذلك الهدف يتضمن تحقيق الاهداف التالية :1- إلقاء الضوء علي التجارة البينية لأهم مجموعات السلع الغذائية.

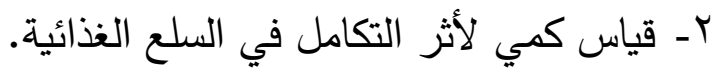
r- (بياس نسبي لأثر التكامل في السلع الغذائية.

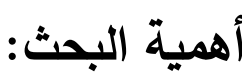
ترجع أهمية الدراسة إلى أهمية الموضوع حيث تناول العديد من الاتفاقيات التجارية بصدد تتمية التجارة البينية وما يمكن أن تعززه من منافع ومكاسب بين الدول الأعضاء. وفى ظل هذه الظروف التي تدعو لتحرير الاقتصاد والعولمة ذادت أهمية التكامل الاقتصادي لبعض دول مجلس الخليج. ومن هنا تبرز أهمية الدراسة في كونها تحلل الواقع الاقتصادي العربي الراهن من خلال بيان تطور حجم الموارد الطبيعية وحجم التجارة العربية البينية لدول التكامل ودورها في تحقيق التكامل الاقتصادي، ومحاولة تحديد المزايا والفوائد التي تنجم عن تحقيق التكامل الاقتصادي ، وكذلك تحديد المشاكل والمعوقات التي تواجه تحقيق التكامل الاقتصادي العربي. المبحث الأول: مفهوم التكامل الاقتصادي: 


\section{أولا: تعريف التكامل الاقتصادي:}

قبل التطرق الي مفهوم التكامل الاقتصادي، لابد من التعريف بمصطلح التكامل وابتدئ استعمالها عام •بrآ في قاموس أكسفورد الانجليزي كلمة التكامل جعل الأجزاء متفرقة كلا متكاملا. ويستخدم مصطلح التكامل لنقل معني التعبير الانجليزي(Integration) اشتقاقا من لفظ (Integer) الذي يعني الثئ المتراكب عضويا في كل لا يتجزأ. هناك اختلاف كبير بين الاقتصاديين حول تحديد مصطلح التكامل الاقتصادي، ويرجع هذا الاختلاف بشكل عام الي التباين في وجهات نظر هولاء الاقتصاديين حول درجة ونوع التعاون الاقتصادي القائم أو المتترح بين الدول المختلفة محل الدراسة علي أساس النظر إليها كوحدات اقتصادية مستقلة ترغب في إقامة شكل أو أخر من أثكال التكامل الاقتصادي. يعرف التكامل علي انه تجميع عدة عناصر منتشرة لتكوين كل واحد جديد، وكبير لتقوية كل موجود وغير مترابط.

ويعرف بيلا يلاسا التكامل الاقتصادي علي أنه عملية وحالة، فبوصفه عملية فإنه يشمل الإجراءات والتدابير التي تؤدي الي إلغاء التمييز بين الواحدات المنتمية الي دول قومية مختلفة، وإذا نظرنا إليه علي أنه حالة فهو يتمثل في انتقاء مختلف صور التثرقة بين اقتصاديات الدول، وانطلاقا من ذلك يذهب هذا الاقتصادي الي اعتبار أن 
اختفاء التمييز أو التدخل الحكومي يعد شرطا منطقيا ومدخلا طبيعيا لتحرير التجارة بين مجموعة الدول الأعضاء في التنظيم التكاملي. ويري جان تتبرجن أن التكامل الاقتصادي كعملية يشتمل علي العديد من الجوانب التي ذكرها بلاسا فيري أنه عبارة عن إيجاد أحسن السبل الأطر للعلاقات الاقتصادية الدولية والسعي لإزلة كافة العقبات والمعوقات أمام هذا التعاون. أما جونار ميرا دال ، فيري أن التكامل لابد وأن يشمل العمل علي زيادة الكفاءة الإنتاجية ضمن التكتل الاقتصادي القائم، وذلك بإعطاء الفرص الاقتصادية المتساوية للأعضاء في هذا التكتل بغض النظر عن سياساتهم. علي أن واقع الأمر يشير الي وجود اتجاهين رئيسين يمكن التميز بينهما. الاتجاه الأول: اتجاه عام يعرف التكامل علي أنه أي شكل من أشكال التعاون، أو التنسيق بين الدول المختلفة دون المساس بسيادة أي منها، وينتقد هذا التعريف لاتساعه، الأمر الذي يجعل العلاقات ذات الطبع التعاوني بمثابة علاقات تكاملية وهو ما يغفل التمييز بين التكامل من ناحية والتعاون والتتسيق من ناحية أخري. الاتجاه الثاني: هو الأكثر تحديدا حيث يعتبر التكامل عملية لتطوير العلاقات بين الدول وصولا الي أشطال جديدة مشتركة بين المؤسسات والتفاعلات التي تؤثر

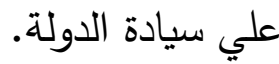


وبشكل عام يمكن النظر الي التكامل علي انه حالة تقود الدول الأعضاء في المنطقة التكاملية الي وضع يتجه نحو التخصيص الأمثل للموارد الاقتصادية، كما يتجه الي إذابة الاقتصاديات بالإجراءات والتدابير الضرورية والكافية لإحداث التغييرات المطلوبة في الهياكل الاقتصادية للدول الأعضاء في المنطقة التكاملية للوصول الي الكيان التكاملي الجديد كهذف نهائي. في حين يري لبعض الآخر أن التكامل الاقتصادي هو: عملية تقارب تدريجية تعمل علي تسهيل تتمية البلدان ذات الأنظمة السياسية والإقتصادية المتجانسة، ولذا يجب أن يكون متتاسقا مع حطط التنمية. ثالثا: أهمية التكامل الاقتصادي:

يلعب التكامل الاقتصادي دورا مهما في حياة البلدان الأعضاء في العملية التكاملية ، إذ تتجلى هذه الأهمية من خلال أهم النقاط التالية :

ا - يمكن للدول المندرجة ضمن إطار العملية التكاملية من الاستفادة من الحجم الكبير للسوق و تجاوز صعوبات ضيق السوق المحلية، حيث" يشجع سوق السوق الكبير على توجيه الاستثمارات توجيها اقتصاديا سليما، و إعادة تكوين الحركة الحرة للسلع ورأس المال، والعمل من دولة لأخرى، و إزالة كل العوائق في هذا المجال حيث يشجع اتساع نطاق السوق على إقامة صناعات ذات حجم اقتصاد تنخف فيها 
تكاليف الإنتاج لارجة تمكن المشروعات من التسويق خارج أسواق الدول الأعضاء.

ז- خلق فرص ممتازة لتوفير مستلزمات التتمية البشرية الغذائية السكنية، الصحية والتعليمية وهذا لإثباع الحاجات الأساسية لثعوب الدول الأعضاء في العملية التكاملية، كما تكتسي التكتلات الاقتصادية الاقليمية أهمية بالغة في عالمنا المعاصر، لأنها تمكن تحقيق الكثير من المكاسب فالتكتل ليس هدفا في حد ذاته إنما هو عبارة عن وسيلة لتحقيق أهداف مختلفة نذكر من بيتها. أ- بأنه أثر خلق التجارة الأثر الإنتاجي للتكامل والذي أشار إليه فاينر ومفاده أن زيادة رفاهية الدول الأعضاءتأتي كنتيجة طبيعية لزيادة إنتاج المشروعات ذات الكفاءة العالية علي حساب المشؤوعات الأقل كفاءة. ب- الأثر الاستهلاكي للتكامل الاقتصادي، حيث يؤدي التكامل الاقتصادي الي زيادة رفاهية المستهلكين عن طريق إحلال السلع رخيصة الثمن محل السلع مرتفعة الثمن وزيادة عدد السلع المتاحة مع تحسين جودتها. ج-يساهم التكامل الاقتصادي في زيادة معدلات التبادل التجاري للدول الأعضاء، كما يؤدي الي زيادة القوة التفاوضية لأنه كلما زاد عدد الأعضاء كلما كانت هناك قوة تفاوضية أفضل مع الدول الأخري ومن ثم فإن حجم تجارتها مع العالم الخارجي سيكون في وضع أفضل، أي أن التكامل الاقتصادي يحسن معدل التبادل التجاري. 
د- تحقيق الوافرات الخارجية نتيجة انتقال عناصر الإنتاج بحرية ودون قيود بين دول الهجموعة، مما يؤدي الي انخفاض التكاليف لمعظم المشروعات نتيجة الاستخدام الأمثل للموارد المتاحة، وتعتبر هذه الوفورات من أهم المكاسب التي تعظم أهمية

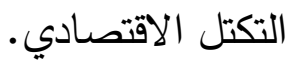
هـ - يعمل التكامل الاقتصادي علي زيادة معدلات النمو الاقتصادي للدول الأعضاء، وذلك كنتيجة طبيعية لزيادة حجم الاستثمارات واختيار أفضل المواقع لها بعدما أصبح في الإمكان إنثاء مشروعات وفق معايير اقتصادية وليس بصورة عشوائية، علاوة علي تثجيع الاستثمارات الأجنبية. كما تبرز أهمية التكامل الاقتصادي في زيادة وتثجيع حجم التجارة بين الدول وذلك نتيجة إلغاء القيود المختلفة سواء كانت جمركية أو إدارية أو أي معوقات أخري، علاوة علي أن التكامل الاقتصادي يجد الدول الأعضاء من التغيرات الفجائية في سياساتها التجارية.

نتائج الدراسة:

المبحث الثاني: التجارة الخارجية البينية لمجموعة السلع الغذائية:

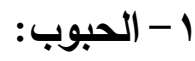

بدراسة وتحليل تطور التجارة الخارجية للحبوب في السعودية خلال الفترة (·. بكما في الجدول رقم (1) يتضح أن المتوسط السنوي لقيمة الصادرات السعودية من الحبوب بلغ نحو 15.3 مليون ، في حين بلغ المتوسط السنوي لقيمة 
وارداتها نحو 4242.8 مليون دولار، كما حقق الميزان التجاري لمجمعة الحبوب خلال فترة الدراسة عجزا سنويا قدر في المتوسط بنحو 4227.5 مليون دولار،. وفي الامارات بلغ المتوسط السنوي لقيمة الصادرات من الحبوب نحو 428.8 مليون دولار ، في حين بلغ المتوسط السنوي لقيمة وارداتها نحو 1496.1 مليون دولار ، كما حقق

الميزان التجاري للحبوب عجزا سنويا قدر في المتوسط بنحو 1067.3 مليون دولار . كما بلغ المتوسط السنوي لقيمة الصادرات من الحبوب في الكويت نحو 33.7مليون دولار في حين بلغ المتوسط السنوي لقيمة واردات الجزائر نحو 495.6 مليون دولار ، كما حقق الميزان التجاري للحبوب عجزا سنويا قدر في المتوسط بنحو

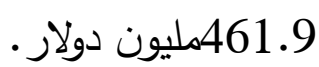

جدول رقم (1) تطور قيمة التجارة الخارجية للحبوب في السعودية والامارت والكويت بالمليون دولار خلال الفترة( 2010-2016)

\begin{tabular}{|c|c|c|c|c|c|c|c|c|c|}
\hline \multicolumn{3}{|c|}{ الكويت } & \multicolumn{3}{|c|}{ الامارات } & \multicolumn{3}{|c|}{ السعودية } & \multirow[t]{2}{*}{ السنوات } \\
\hline الميزان & الواردات & الصادرات & الميزان & الواردات & الصادرات & الميزان & الواردات & الصادرات & \\
\hline ऍ^^.૫- & $r q . . v$ & Y.l & $1.19 .0-$ & 1709.9 & $o v \cdot . \varepsilon$ & EIYI.0- & צחו & $1 \leq .0$ & 2010 \\
\hline 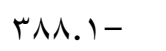 & $r q . . r$ & Y.l & IYT.O- & $|\wedge| Y . \wedge$ & $00 . r$ & $\varepsilon r q \vee . q-$ & $\leqslant \leqslant 1 \leqslant .0$ & 17.7 & 2011 \\
\hline$\varepsilon \Sigma 1 . \wedge-$ & $\leq 7 \wedge .1$ & r.r & $997-$ & ع.. & Tצ. & $\Sigma q ৭ \vee . \vee-$ & $0.17 . \varepsilon$ & $1 \Lambda . V$ & 2012 \\
\hline$\varepsilon \leqslant Y .0-$ & $\sum \vee \wedge .7$ & r..l & $1 \cdot V Y . \varepsilon-$ & V. . & YOA.r & $7.07 .0-$ & $7 . V \varepsilon . V$ & $1 \Lambda . r$ & 2013 \\
\hline ory.z- & $0 \vee 0.9$ & rq.० & IIVT.A- & $\mid \leq r Y .1$ & rOA.r & Oor..r- & $00 \leqslant \varepsilon . \varepsilon$ & $1 \varepsilon . r$ & 2014 \\
\hline $07 \leqslant-$ & TIT.T & $\leq 9.7$ & ITH . & 1090 & Yтะ.T & $\varepsilon \cdot \Lambda \cdot . r-$ & $\varepsilon .97 . \varepsilon$ & 17.1 & 2015 \\
\hline$\sum \Sigma \neg . \wedge-$ & $0 . Y_{.1}$ & 00.1 & NTo.0- & IEYY.A & ONV.r & TYTT- & YYVE.A & IY.A & 2016 \\
\hline
\end{tabular}




\begin{tabular}{|c|c|c|c|c|c|c|c|c|c|}
\hline 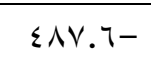 & $0 \leqslant T . Y$ & 01.7 & 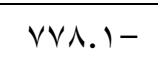 & 1500.7 & OVV.O & rTVT.q- & $r T \wedge 0.0$ & 11.7 & 2017 \\
\hline-461.9 & 495.6 & 33.7 & -1067.3 & 1496.1 & 428.8 & -4227.5 & 4242.8 & 15.3 & المتوسط \\
\hline
\end{tabular}

ألعصداد : جمعت وحسبت من: جامعة الدول العربية، المنظمة العربية للتمية الزراعية، الكتاب السنوي للإحصاءات الزراعية العربية، الخرطوم،

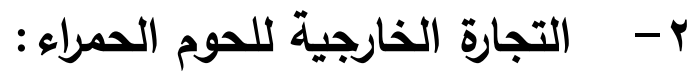

طبقا لما هو مبين بالجدول رقم(2) بدراسة وتحليل تطور التجارة الخارجية

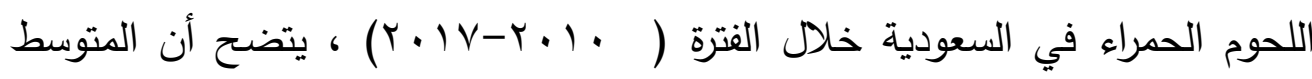
السنوي لقيمة الصادرات السعودية من اللحوم الحمراء بلغ نحو108.4 مليون ، في حين بلغ المتوسط السنوي لقيمة وارداتها نحو 1250.35 مليون دولار، كما حقق الديزان التجاري لمجموعة الحبوب خلال فترة الدراسة عجزا سنويا قدر في المتوسط بنحو 1141.5 مليون دولار،. وفي الامارات بلغ المتوسط السنوي لقيمة الصادرات من اللحوم الحمراء نحو 61.14 مليون دولار ، في حين بلغ المتوسط السنوي لقيمة وارداتها نحو 1136.2 مليون دولار، كما حقق الميزان التجاري للحبوب عجزا سنويا قدر في المتوسط بنحو 1075.1 مليون دولار.

جدول رقم (ץ) تطور قيمة التجارة الخارجية اللحوم في السعودية والامارت والكويت بالمليون دولار خلال الفترة (2010-2016)

\begin{tabular}{|c|c|c|c|c|c|c|c|c|c|}
\hline \multicolumn{3}{|c|}{ الكويت } & \multicolumn{3}{|c|}{ الامارات } & \multicolumn{3}{|c|}{ السعودية } & \multirow[t]{2}{*}{ لسنوات } \\
\hline الميزان & الواردات & الصادرات & الميزان & الواردات & الصادرات & الميزان & الواردات & الصادرات & \\
\hline 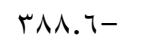 & $r q . . v$ & r. 1 & $1019.0-$ & 1709.9 & $v \cdot . \varepsilon$ & $11 K 1.0-$ & $11 \pi 4$ & $1 \leqslant .0$ & 2010 \\
\hline
\end{tabular}




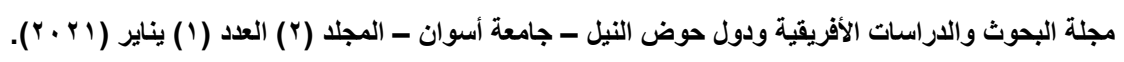

\begin{tabular}{|c|c|c|c|c|c|c|c|c|c|}
\hline 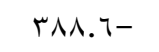 & $r q . . v$ & r.l & IVTY.O- & MIY.A & $0 . . r$ & 1 1T9V.9- & $1 \leqslant 1 \leqslant .0$ & 17.7 & 2011 \\
\hline IVV.A- & $11 . .0$ & r.V & $r \leq Y . \varepsilon-$ & $r .0 .9$ & Tr.o & $77 V .0-$ & $\Lambda \cdot 1 . r$ & & 2012 \\
\hline $119.7-$ & $190 . \mathrm{V}$ & 7.1 & rrr.q- & $r .0 . V$ & $\wedge 1 . \wedge$ & $741.9-$ & VฯA.r & $99 . \%$ & 2013 \\
\hline $1 \leqslant V .00-$ & $1 \leq 9.7$ & r..o & rrq.7- & $r .0 . V$ & 74.1 & vor.A- & ATr.V & $1 \cdot 1.9$ & 2014 \\
\hline ors.V- & 000.0 & $r \cdot . \Lambda$ & $10 \mathrm{V0.V-}$ & $17 r \leq .1$ & $\varepsilon \wedge . \varepsilon$ & $1910.9-$ & $r \cdot 10.2$ & 179.0 & 2015 \\
\hline $70.7-$ & $V Y .0$ & 11.9 & $\begin{array}{c}- \\
\text { lork.AT }\end{array}$ & 1010.9 & or.. \& & $11 \wedge v .0-$ & $1 T \leq 7 . \varepsilon$ & 101.9 & 2016 \\
\hline$\varepsilon \leq \leqslant-$ & $\leqslant 07.0$ & IT.O & $1 \leqslant r \leqslant-$ & $1 \leqslant \wedge 9.7$ & 00.7 & $1 \leq r Y .0-$ & 1011.5 & 170.9 & 2017 \\
\hline-292.06 & 299.5 & 7.53 & -1075.1 & 1136.2 & 61.14 & -1141.9 & 1250.35 & 108.4 & المتوسط \\
\hline
\end{tabular}

المصدر : جمعت وحسبت من: جامعة الدول العربية، المنظمة العربية للتتمية الزراعية، الكتاب السنوي للإحصاءات الزراعية العربية، الخرطوم،

أعداد مختلفة.

كما بلغ المتوسط السنوي لقيمة الصادرات من اللحوم الحمراء في الكويت نحو

7.53 مليون دولار في حين بلغ المتوسط السنوي لقيمة واردات الجزائر نحو 299.5

مليون دولار، كما حقق الميزان التجاري للحبوب عجزا سنويا قدر في المتوسط بنحو

292.06مليون دولار .

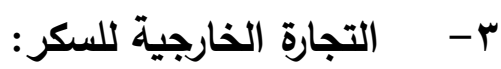

بدراسة وتحليل تطور التجارة الخارجية السكر في السعودية خلال الفترة

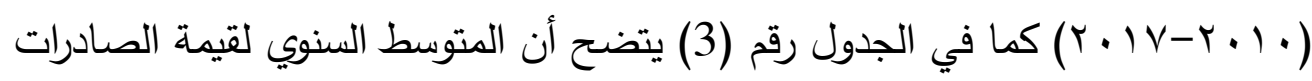

السعودية من السكر بلغ نحو210.7 مليون دولار ، في حين بلغ المتوسط السنوي

لقيمة وارداتها نحو 639.5 مليون دولار، كما حقق الميزان التجاري السكر خلال فترة

الدراسة عجزا سنويا قدر في المتوسط بنحو 428.7 مليون دولار، وفي الامارات بلغ

المتوسط السنوي لقيمة الصادرات من السكر نحو 578.4 مليون دولار ، في حين بلغ 
المتوسط السنوي لقيمة وارداتها نحو 710.15 مليون دولار، كما حقق الديزان التجاري للسكر عجزا سنويا قدر في المتوسط بنحو 133.75 مليون دولار. كما بلغ المتوسط السنوي لقيمة الصادرات من السكر في الكويت نحو 7.35مليون دولار في حين بلغ المتوسط السنوي لقيمة واردات نحو 93.11 مليون دولار، كما حقق الميزان التجاري للسكر عجزا سنويا قدر في المتوسط بنحو

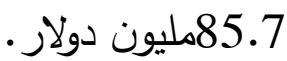

\section{ع - التجارة الخارجية للزيوت:}

بدراسة وتحليل تطور التجارة الخارجية للزيوت في السعودية خلال الفترة

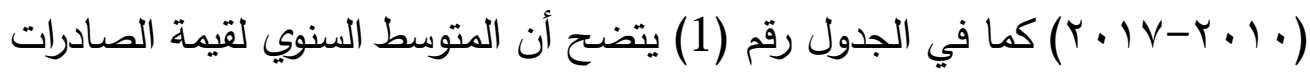
السعودية من الزيوت بلغ نحو253.03 مليون دولار ، في حين بلغ المتوسط السنوي لقيمة وارداتها نحو 492.3 مليون دولار، كما حقق الديزان التجاري لمجموعة الزيوت خلال فترة الدراسة عجزا سنويا قدر في الدتوسط بنحو 239.3 مليون دولار،. وفي الامارات بلغ المتوسط السنوي لقيمة الصادرات من الزيوت نحو 215.1 مليون دولار ، في حين بلغ المتوسط السنوي لقيمة وارداتها نحو 520.55 مليون دولار، كما حقق الميزان التجاري للزيوت عجزا سنويا قدر في المتوسط بنحو 305.45 مليون دولار. كما بلغ المتوسط السنوي لقيمة الصادرات من الزيوت في الكويت نحو 6.66مليون دولار في حين بلغ المتوسط السنوي لقيمة وارداتها نحو 135.93 مليون 
دولار، كما حقق الميزان التجاري للزيوت عجزا سنويا قدر في المتوسط بنحو

• 129.27مليون دولار

جدول رقم(r) تطور قيمة التجارة الخارجية للسكر في السعودية والامارت والكويت

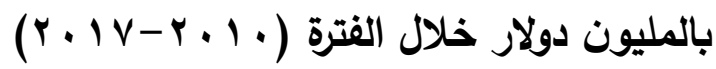

\begin{tabular}{|c|c|c|c|c|c|c|c|c|c|}
\hline \multicolumn{3}{|c|}{ الكويت } & \multicolumn{3}{|c|}{ الامارات } & \multicolumn{3}{|c|}{ السعودية } & \multirow[t]{2}{*}{ السنوات } \\
\hline الميزان & الواردات & ت الصادرا & الميزان & الواردات & تالصادرا & الميزان & الواردات & تالصادرا & \\
\hline or.11- & Or.r &..$r$ & $r . \tau . r$ & ros.7 & $77 . .9$ & $\begin{array}{r}- \\
v \cdot v .7\end{array}$ & 199.1 & 191.0 & 2010 \\
\hline or.l1- & Or.r &..$r$ & YTY.O & $\leq \leqslant \vee .1$ & $\vee \cdot 9.7$ & $\begin{array}{r}- \\
\text { - }\end{array}$ & 人ั9.1 & $r \ldots . \wedge$ & 2011 \\
\hline $11 \leqslant .7-$ & 1 1Y. & 11.0 & 191.1- & $1171 . r$ & $r v \cdot . l$ & $\begin{array}{r}- \\
\varepsilon r v . \varepsilon\end{array}$ & $V \cdot 9 . r$ & rVI.A & 2012 \\
\hline$V \leqslant .7 V-$ & vo.1 &..$\leqslant r$ & 79Y.1- & $1101 . \mathrm{V}$ & $\leq 09.7$ & - & $01 . .1$ & $198 . V$ & 2013 \\
\hline 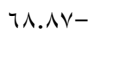 & $79 . r$ & . & Arr.q- & IrAT.o & $\leq 09.7$ & - & $r \leq . . T$ & $11 \cdot .0$ & 2014 \\
\hline $11 \leqslant .1-$ & ITr.o & $11 . \varepsilon$ & $\mid V \leq . \wedge$ & $\varepsilon r 1.1$ & 7.0 .9 & $\begin{array}{r}- \\
\text { ror.r }\end{array}$ & 1०ᄉ. $\varepsilon$ & Y.r.r & 2015 \\
\hline $1.7 .1-$ & 111.2 & $1 Y .7$ & rol.r & 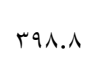 & vo..l & $\begin{array}{r}- \\
\text { rTV.r }\end{array}$ & $0 . T . \varepsilon$ & 1 & 2016 \\
\hline 1.r. $\leqslant-$ & 111.1 & $10 . \varepsilon$ & $r \leq r . r$ & sor.r & $790 . \varepsilon$ & $\begin{array}{r}- \\
\text { ro. } 9\end{array}$ & 0Y7.0 & rVo.T & 2017 \\
\hline-85.7 & 93.11 & 7.35 & -133.75 & 710.15 & 576.4 & $\begin{array}{c}- \\
428.7\end{array}$ & 639.5 & 210.7 & المتوسط \\
\hline
\end{tabular}

المصدر : جمعت وحسبت من: جامعة الدول العربية، المنظمة العربية للتمية الزراعية، الكتاب السنوي للإحصاءات الزراعية العربية، الخرطوم، أعداد مختلفة.

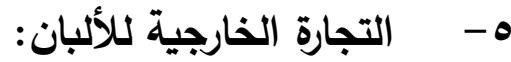

بدراسة وتحليل تطور التجارة الخارجية للالبان في السعودية خلال الفترة )

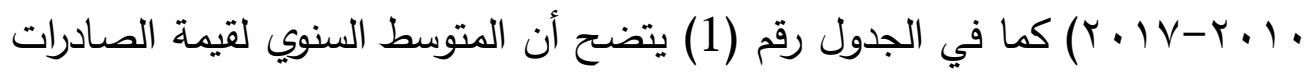

السعودية من الالبان بلغ نحو778.6 مليون دولار، في حين بلغ المتوسط السنوي 
لقيمة وارداتها نحو 1584.7 مليون دولار، كما حقق الميزان التجاري للالبان خلال فترة الدراسة عجزا سنويا قدر في المتوسط بنحو 806.1 مليون دولار ، وفي الامارات بلغ المتوسط السنوي لقيمة الصادرات من الألبان نحو 407.23 مليون دولار ، في حين بلغ المتوسط السنوي لقيمة وارداتها نحو 1070.2 مليون دولار، كما حقق الميزان التجاري للالبان عجزا سنويا قدر في المتوسط بنحو 662.9 مليون دولار . كما بلغ المتوسط السنوي لقيمة الصادرات من الالبان في الكويت نحو 84.53مليون دولار في حين بلغ المتوسط السنوي لقيمة وارداتها نحو 380.5 مليون دولار، كما حقق الميزان التجاري للالبان عجزا سنويا قدر في المتوسط بنحو

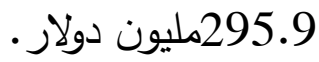

جدول رقم (ع) تطور قيمة التجارة الخارجية للزيوت في السعودية والامارت والكويت بالمليون دولار خلال الفترة ( 2010-2017)

\begin{tabular}{|c|c|c|c|c|c|c|c|c|c|}
\hline \multicolumn{3}{|c|}{ الكويت } & \multicolumn{3}{|c|}{ الامارات } & \multicolumn{3}{|c|}{ السعودية } & \multirow[t]{2}{*}{ السنوات } \\
\hline الميزان & الواردات & الصادرات & الميزان & الواردات & الصادرات & الميزان & الواردات & الصادرات & \\
\hline 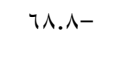 & vi.r & 9.0 & -r.r.r- & $\varepsilon 9 \leqslant . A$ & $17 \varepsilon .0$ & $T \leqslant . V-$ & $r \leq 0.0$ & $1 \wedge . .1$ & 2010 \\
\hline ТА.A- & vı.r & 9.0 & $r r . . r-$ & $\varepsilon 9$ \&.. & $17 \varepsilon .0$ & $\begin{array}{c}- \\
17 V . A\end{array}$ & $\varepsilon \vee \cdot . q$ & $r \cdot r \cdot 1$ & 2011 \\
\hline $1 V V .1-$ & $1 \wedge . .1$ & r.v & rT..r- & $\varepsilon 9 \leqslant . \wedge$ & $17 \leqslant .0$ & $\begin{array}{c}- \\
710.1\end{array}$ & 9Y0.\& & $r 1 . r$ & 2012 \\
\hline $1 \times 4.19-$ & Irq.r & r.11 & rr..r- & $\varepsilon 9 \varepsilon . \wedge$ & $17 \leqslant .0$ & $\begin{array}{c}- \\
\text { oor.1 }\end{array}$ & $\lambda \backslash \leqslant . \lambda$ & YभI.V & 2013 \\
\hline $1.9 .04-$ & $11 \% .1$ & r.OV & r.r.r- & $\varepsilon 9 \varepsilon . \wedge$ & $17 \varepsilon .0$ & $\begin{array}{c}- \\
\leq 71.9\end{array}$ & $v \cdot 0.9$ & $r \varepsilon \varepsilon$ & 2014 \\
\hline $170 . \leqslant 9-$ & IVY.O & v.. & rov.V- & oor.r & rq६. & r..气- & $r 90.9$ & YYะ.O & 2015 \\
\hline $171.7-$ & $1 v . . r$ & A.. $\mathrm{V}$ & $r V_{0} . \varepsilon-$ & - & rq५.० & $11-$ & rro.1 & rY $\{.1$ & 2016 \\
\hline
\end{tabular}




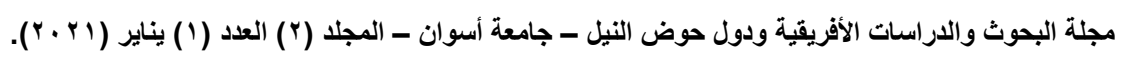

\begin{tabular}{|c|c|c|c|c|c|c|c|c|c|}
\hline $107 . \mathrm{V}-$ & 170.9 & Q. . & YY & OV & $r$ r..r & $9 . \wedge-$ & $r \leqslant 0.7$ & rro.A & 2017 \\
\hline-129.27 & 135.93 & 6.66 & -305.45 & 520.55 & 215.1 & $\begin{array}{c}- \\
239.3\end{array}$ & 492.3 & 253.03 & المتوسط \\
\hline
\end{tabular}

المصدر: جمعت وحسبت من: جامعة الدول العربية، المنظمة العربية للتمية الزراعية، الكتاب السنوي للإحصاءات الززاعية العربية، الخرطوم،

أعداد مختلفة.

ثانيا: فرص واثز التوجه نحو التكامل علي التجارة البينية لأهم السلع الغذائية

ان توجيه صادرات الحاصلات الزراعية مع العالم الخارجي إلي صادرات

زراعية بينية يساعد علي زيادة حجم التجارة البينية للسعودية و الامارات و الكويت

وتقليل اعتمادها علي الخارج مما قد يساعد في تحقيق درجة أفضل

جدول رقم (0) تطور قيمة التجارة الخارجية للألبان في السعودية والامارت والكويت

بالمليون دولار خلال الفترة ( 2010- 2017)

\begin{tabular}{|c|c|c|c|c|c|c|c|c|c|}
\hline \multicolumn{3}{|c|}{ الكويت } & \multicolumn{3}{|c|}{ الامارات } & \multicolumn{3}{|c|}{ السعودية } & \multirow[t]{2}{*}{ السنوات } \\
\hline الميزان & الواردات & ت الصادرا & الميزان & الواردات & تالصادرا & الميزان & الواردات & تالصادرا & \\
\hline$|r \cdot r|-$ & 151.9 & 1.09 & $\varepsilon \mid r .1-$ & $\tau \leqslant \Lambda . \leqslant$ & rrד.r & $\begin{array}{c}- \\
1 \ldots 9.7\end{array}$ & $\begin{array}{l}1111 . \\
\varepsilon\end{array}$ & $1 \cdot \wedge .1$ & 2010 \\
\hline 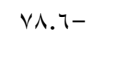 & 151.9 & or.r & 纟ภr.r- & $v 9 . .0$ & $r \cdot v \cdot r$ & $971.7-$ & $\begin{array}{c}1.1 . \\
r\end{array}$ & 111.7 & 2011 \\
\hline ror.A- & TrT.E & 79.7 & ง9ะ.9- & |r|T.A & r11.9 & rrr.q- & $\begin{array}{c}\text { Irr. } \\
r\end{array}$ & $9 \wedge$ १ั. & 2012 \\
\hline Y9०- & TาT.\& & $T V . \varepsilon$ & $0 \leq .7-$ & 914.7 & TVY & $110 .\{-$ & $\begin{array}{c}17 \leq r . \\
V\end{array}$ & NYA.r & 2013 \\
\hline r9o- & rTr.s & $T V . \varepsilon$ & $\varepsilon \wedge \vee .{ }^{\prime}-$ & 914.7 & $\varepsilon r 0 . r$ & $\begin{array}{c}- \\
\text { irvı.o }\end{array}$ & $\begin{array}{c}r \cdot \lambda l . \\
0\end{array}$ &.$v \cdot r$ & 2014 \\
\hline$\leqslant \vee \leqslant . \wedge-$ & Tा纟.r & $1 \% 9.0$ & A) .r. & $1 \leq \leqslant \leq .0$ & Tr人.r & NYI- & $\begin{array}{c}\text { IqrV. } \\
r\end{array}$ & 11.4 .5 & 2015 \\
\hline 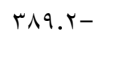 & or $\leq .0$ & iro.r & งr৯.v- & IY $97 . V$ & $\leqslant 0 \wedge$ & $7.9-$ & $\begin{array}{c}\mid v \leq r . \\
1\end{array}$ & $11 r \varepsilon .1$ & 2016 \\
\hline 纟or.1- & $09 \varepsilon . r$ & $1 \leq r . r$ & Nr..o- & $1 r \leqslant r .0$ & 011 & TIY.A- & אוtr. & 1Yo..s & 2017 \\
\hline
\end{tabular}




\begin{tabular}{|r|r|r|r|r|r|r|r|r|c|}
\hline-295.9 & 380.5 & 84.53 & -662.9 & 1070.2 & 407.23 & -806.1 & 1584. & 778.6 & 7 \\
\end{tabular}

المصدر: جمعت وحسبت من: جامعة الدول العربية، المنظمة العربية للتمية الزراعية، الكتاب السنوي للإحصاءات الزراعية العربية، الخرطوم، أعداد مختلفة.

- - أثر التكامل علي التجارة البينية للحبوب:

يتضح من البيانات الواردة بالجدول رقم (T) انه يمكن زيادة حجم التجارة

البينية لسعودية والامارات من الحبوب عن طريق تحويل صادراتهم الخارجية الي بينية، الأمر الذي يترتب عليه زيادة صادرات الحبوب بين سعودية والامارات خلال الفترة (2014 - 2017 ) بنحو 484.74 مليون دولار، وبمقتضى ذلك تعتبر الامارات أكثر القادرين علي المساهمة في زيادة تجارة الصادرات البينية الحالية حيث يمكن أن تساهم في ضوء الطاقة الاستيعابية بنحو 97.4\% من تلك الزيادة وتساهم السعودية بنحو 2.6\%، بينما يمكن زيادة صادرات الحبوب بين السعودية و الكويت بنحو 59.9 مليون دولار، وبالتالي تساهم الكويت في زيادة الصادرات البينية في ضوء الطاقة الاستيعابية بنحو 84.6\% ، وتساهم السعودية بنحو 15.4\% جدول رقم (؟) أثر التكامل بين السعودية وكل من والامارت والكويت علي

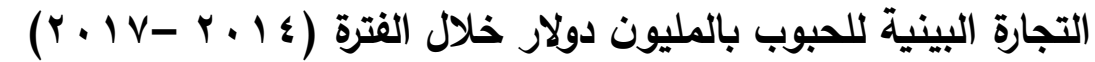

\begin{tabular}{|c|c|c|c|c|c|c|c|c|}
\hline \multicolumn{2}{|c|}{ أثر التكامل } & \multicolumn{3}{|c|}{ قيمة تجارة البينية } & \multicolumn{3}{|c|}{ قيمة تجارة الكلية } & الدولة \\
\hline \%من إجمالي الأثر & القيمة & حجم التجارة & واردات & صادرات & حجم التجارة & واردات & صادرات & \\
\hline 2.6 & 12.79 & r..99- & $\varepsilon \cdot . V$ & $\because v \cdot v$ & $r q .0 . \varepsilon-$ & r 911.9 & 15.0 & السعودية \\
\hline 97.4 & 471.95 & $r .10$ & $1 . r V$ & $\leq . \leq r$ & $911 . \leqslant r-$ & $1 \leqslant 0 V .1$ & $\begin{array}{c}\varepsilon V T . r \\
V\end{array}$ & الامارات \\
\hline 100 & 484.74 & 36.84 & 41.97 & 5.427 & $\begin{array}{c}- \\
3723.83\end{array}$ & 4376.7 & 489.9 & الاجمالي \\
\hline \%من إجمالي الأثر & القيمة & حجم التجارة & واردات & صادرات & حجم التجارة & واردات & صادرات & \\
\hline 15.4 & 9.23 & $\cdot .719$ & $r .70$ & $\varepsilon . r V$ & rq.0.5- & r911.9 & $1 r .0$ & السعودية \\
\hline
\end{tabular}




\begin{tabular}{|c|c|c|c|c|c|c|c|c|}
\hline 84.6 & 50.67 & r.l $\leq$ & 1.79 & r.Ar & $\leqslant 99 . \leqslant \vee-$ & oor.9v & $0 \leqslant .0$ & الكويت \\
\hline 100 & 59.9 & 2.76 & 5.34 & 8.1 & 3404.87 & 3472.87 & 68 & الاجمالي \\
\hline
\end{tabular}

\section{r-أثر التكامل علي التجارة البينية للحوم الحمراء:}

يتضح من البيانات الواردة بالجدول رقم (7) انه يمكن زيادة حجم التجارة البينية

لسعودية والامارات من اللحوم الحمراء عن طريق تحويل صادراتهم الخارجية الي بينية، الأمر الذي يترتب عليه زيادة صادرات اللحوم الحمراء بين سعودية والامارات خلال الفترة ( 2014 - 2017 ) بنحو 41.11 مليون دولار ، وبمقتضى ذلك تعتبر السعودية أكثر القادرين علي المساهمة في زيادة تجارة الصادرات البينية الحالية حيث يمكن أن تساهم في ضوء الطاقة الاستيعابية بنحو 52.1\% من تلك الزيادة وتساهم الامارات بنحو 47.9\%، بينما يمكن زيادة صادرات اللحوم الحمراء بين السعودية و الكويت بنحو 22.3 مليون دولار ، وبالتالي تساهم السعودية في زيادة الصادرات البينية في ضوء الطاقة الاستيعابية

$$
\text { بنحو 79.4\% ، وتساهم الكويت بنحو 20.6\%. }
$$

جدول رقم (V) أثر التكامل بين السعودية وكل من والامارت والكويت علي

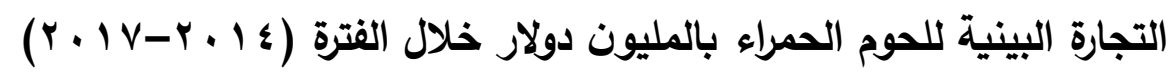

\begin{tabular}{|c|c|c|c|c|c|c|c|c|}
\hline \multicolumn{2}{|c|}{ أثر التكامل } & \multicolumn{3}{|c|}{ قيمة تجارة البينية } & \multicolumn{3}{|c|}{ قيمة تجارة الكلية } & الدولة \\
\hline \% إأجمالي & القيمة & التجارة & واردات & صLادرات & التجارة & واردات & صادرات & \\
\hline 52.1 & 21.4 & 22.2 & 17.4 & 4.8 & 3932.1 & 3905.9 & 26.2 & السعودية \\
\hline 47.9 & 19.71 & 48.99 & 48.1 & 0.89 & 1562.6 & 1542 & 20.6 & الامارات \\
\hline 100 & 41.11 & $71-19$ & 65.5 & 4.889 & 5494.7 & 5447.9 & 46.8 & الاجمالي \\
\hline من إجمالي & القيمة & حجم & واردات & صادرات & حجم & واردات & صادرات & \\
\hline
\end{tabular}




\begin{tabular}{|c|c|c|c|c|c|c|c|c|}
\hline \% الأثر & & التجارة & & & التجارة & & & \\
\hline 79.4 & 17.7 & 10.1 & 1.6 & 8.5 & 3932.1 & 3905.9 & 26.2 & السعودية \\
\hline 20.6 & 4.6 & 36.3 & 29.6 & 6.7 & 613.6 & 602.3 & 11.3 & الكوبت \\
\hline 100 & 22.3 & 46.4 & 31.2 & 15.2 & 4545.7 & 4508.2 & 37.5 & الاجمالي \\
\hline
\end{tabular}

المصدر: جمعت وحسبت من: جامعة الدول العربية، المنظمة العربية للتمية الزراعية، الكتاب السنوي للإحصاءات الزراعية العربية، الخرطوم،

أعداد مختلفة.

\section{r- - أثر التكامل علي التجارة البينية للسكر:}

يتضح من البيانات الواردة بالجدول رقم (8) انه يمكن زيادة حجم التجارة البينية

لسعودية والامارات من السكر عن طريق تحويل صادراتهم الخارجية الي بينية، الأمر الذي

يترتب عليه زيادة صادرات السكر بين سعودية والامارات خلال الفترة ( 2014 -

2017) بنحو 844.2 مليون دولار ، وبمقتضى ذلك تعتبر الامارات أكثر القادرين علي المساهمة في زيادة تجارة الصادرات البينية الحالية حيث يمكن أن تساهم في ضوء الطاقة الاستيعابية بنحو 74.1\% من تلك الزيادة وتساهم السعودية بنحو 25.9\%، بينما يمكن زيادة صادرات السكر بين السعودية و الكويت بنحو 230.1 مليون دولار ، وبالتالي تساهم السعودية في زيادة الصادرات البينية في ضوء الطاقة الاستيعابية بنحو 95.1\% ، وتساهم

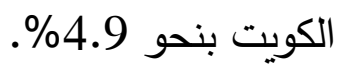

جدول رقم (^) أثر التكامل بين السعودية وكل من والامارت والكويت علي

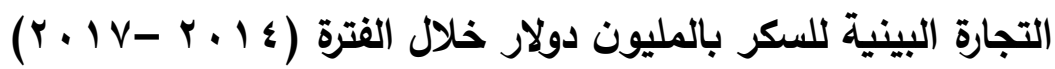

\begin{tabular}{|c|c|c|c|c|c|c|c|c|}
\hline \multicolumn{2}{|c|}{ أثر التكامل } & \multicolumn{3}{|c|}{ قيمة تجارة البينية } & \multicolumn{3}{|c|}{ قيمة تجارة الكلية } & الدولة \\
\hline \% إأجمالي & القيمة & التجارة & واردات & صادرات & التجارة & واردات & صادرات & \\
\hline 25.9 & 218.3 & 140.4 & 129.6 & 10.8 & 887.1 & 658 & 229.1 & السعودية \\
\hline
\end{tabular}




\begin{tabular}{|c|c|c|c|c|c|c|c|c|}
\hline 74.1 & 625.9 & 28.7 & 10.6 & 18.1 & 1348.5 & 704.5 & 644 & الامارات \\
\hline 100 & 844.2 & 169.1 & 140.2 & 28.9 & 2235.6 & 1362.5 & 873.1 & الاجمالي \\
\hline \% من إجمالي & القيمة & التجارة & واردات & صادرات & التجارة & واردات & صادرات & \\
\hline 95.1 & 218.6 & 13 & 2.5 & 10.5 & 887.1 & 658 & 229.1 & السعودية \\
\hline 4.9 & 11.5 & 14.6 & 11.3 & 3.3 & 138.1 & 123.3 & 14.8 & الكويت \\
\hline 100 & 230.1 & 27.6 & 13.8 & 13.8 & 1025.2 & 781.3 & 243.9 & الاجمالي \\
\hline
\end{tabular}

المصدر: جمعت وحسبت من: جامعة الدول العربية، المنظمة العربية للتتمية الزراعية، الكتاب السنوي للإحصاءات الزراعية العببية، الخرطوم، أعداد مختلفة.

أثر التكامل علي التجارة البينية للزيوت:

يتضح من البيانات الواردة بالجدول رقم (9) انه يمكن زيادة حجم التجارة البينية

لسعودية والامارات من الزيوت عن طريق تحويل صادراتهم الخارجية الي بينية، الأمر الذي يترتب عليه زيادة صادرات الزيوت بين سعودية والامارات خلال الفترة ( 2014 2017) بنحو 494.5 مليون دولار، وبمقتضى ذلك تعتبر الامارات أكثر القادرين علي المساهمة في زيادة تجارة الصادرات البينية الحالية حيث يمكن أن تساهم في ضوء الطاقة الاستيعابية بنحو 62.6\% من تلك الزيادة وتساهم السعودية بنحو 37.4\%، بينما يمكن زيادة صادرات الزيوت بين السعودية و الكويت بنحو 239.89 مليون دولار، وبالتالي تساهم السعودية في زيادة الصادرات البينية في ضوء الطاقة الاستيعابية بنحو 98\% ،

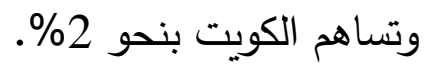

جدول رقم (9) أثر التكامل بين السعودية وكل من والامارت والكويت علي 


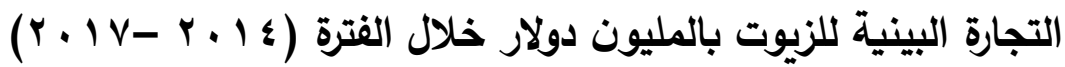

\begin{tabular}{|c|c|c|c|c|c|c|c|c|}
\hline \multicolumn{2}{|c|}{ أثر التكامل } & \multicolumn{3}{|c|}{ قيمة تجارة البينية } & \multicolumn{3}{|c|}{ قيمة تجارة الكلية } & الدولة \\
\hline من إجمالي & القيمة & التجارة & واردات & صادرات & التجارة & واردات & صادرات & \\
\hline 37.4 & 184.7 & 129.4 & 62.8 & 66.6 & 1090.1 & 838.8 & 251.3 & السعودية \\
\hline 62.6 & 309.8 & 98.5 & 79.6 & 18.9 & 928.9 & 600.2 & 328.7 & الامارات \\
\hline 100 & 494.5 & 227.9 & 142.4 & 85.5 & 2019 & 1439 & 580 & الاجمالي \\
\hline من إجمالي & القيمة & التجارة & واردات & صادرات & التجارة & واردات & صادرات & \\
\hline 98 & 235.1 & 18.9 & 2.7 & 16.2 & 1090.1 & 838.8 & 251.3 & السعودية \\
\hline 2 & 4.79 & 21 & 18.2 & 2.8 & 186.99 & 179.4 & 7.59 & الكويت \\
\hline 100 & 239.89 & 39.9 & 20.9 & 19 & 1277.09 & 1018.2 & $\begin{array}{c}258.8 \\
9\end{array}$ & الاجمالي \\
\hline
\end{tabular}

المصدر: جمعت وحسبت من: جامعة الدول العربية، المنظمة العربية للتمية الزراعية، الكتاب السنوي للإحصاءات الزراعية العربية، الخرطوم،

ع - أثر التكامل علي التجارة البينية للألبان:

يتضح من البيانات الواردة بالجدول رقم (10) انه يمكن زيادة حجم التجارة البينية

لسعودية والامارات من الالبان عن طريق تحويل صادراتهم الخارجية الي بينية، الأمر الذي يترتب عليه زيادة صادرات الالبان بين سعودية والامارات خلا الفترة ( 2014 2017) بنحو 1135 مليون دولار، وبمقتضى ذلك تعتبر السعودية أكثر القادرين علي المساهمة في زيادة تجارة الصادرات البينية الحالية حيث يمكن أن تساهم في ضوء الطاقة الاستيعابية بنحو 68.6\% من تلك الزيادة وتساهم الامارات بنحو 31.4\%، بينما يمكن زيادة صادرات الألبان بين السعودية و الكويت بنحو 1015.8 مليون دولار، وبالتالي 
تساهم السعودية في زيادة الصادرات البينية في ضوء الطاقة الاستيعابية بنحو 89.4\% ،

$$
\text { وتساهم الكويت بنحو 10.6\%. }
$$

جدول رقم ( • 1) أثر التكامل بين السعودية وكل من والامارت والكويت علي

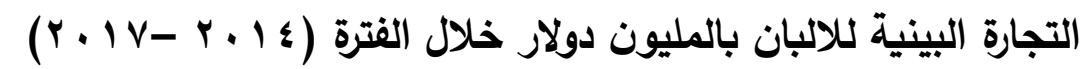

\begin{tabular}{|c|c|c|c|c|c|c|c|c|}
\hline \multicolumn{2}{|c|}{ أثر التكامل } & \multicolumn{3}{|c|}{ قيمة تجارة البينية } & \multicolumn{3}{|c|}{ قيمة تجارة الكلية } & الدولة \\
\hline \%من إجمالي الأثر & القيمة & حجم التجارة & واردات & صادرات & حجم التجارة & واردات & صادرات & \\
\hline 68.6 & 778.2 & 537.6 & 230.6 & 307 & 3095.4 & 2010.2 & 1085.2 & السعودية \\
\hline 31.4 & 356.8 & 505 & 316.6 & 188.4 & 2057.9 & 1512.7 & 545.2 & الامارات \\
\hline 100 & 1135 & 1042.6 & 547.2 & 495.4 & 5153.3 & 3522.9 & 1630.4 & الاجمالي \\
\hline \%من إجمالي الأثر & القيمة & حجم التجارة & واردات & صادرات & حجم التجارة & واردات & صادرات & \\
\hline 89.4 & 907.8 & 204.3 & 26.9 & 177.4 & 3095.4 & 2010.2 & 1085.2 & السعودية \\
\hline 10.6 & 108 & 225.3 & 191.6 & 33.7 & 722.3 & 580.6 & 141.7 & الكويت \\
\hline 100 & 1015.8 & 429.6 & 218.5 & 211.1 & 3817.7 & 2590.8 & 1226.9 & الاجمالي \\
\hline
\end{tabular}

المصدر: جمعت وحسبت من: جامعة الدول العربية، المنظمة العربية للتمية الزراعية، الكتاب السنوي للإحصاءات الزراعية العربية، الخرطوم، أعداد مختلفة. 


\section{الـــراجع}

(1) إكرام عبد الرحيم، التحديات المستقبلية للتكتل الاقتصادي العربي ، القاهرة، مكتبة

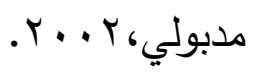

(Y) عبد الرحمن تيشوري، التكامل الاقتصادي العربي في مواجهة التحديات، مقال

$$
\text { اقتصادي منشور علي التالي، www.rezgar.com. }
$$

(r) بوشول السعيد، واقع التكامل الاقتصادي لدول مجلس الخليج العربية وآفاقه، رسالة ماجستير،قسم الاقتصاد و علوم التسيير،كلية الحقوق والعلوم الاقتصادية، جامعة

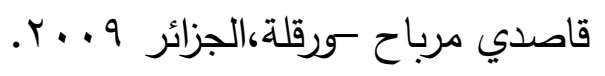

(§) عبد الرحمن تيشوري، التكامل الاقتصادي العربي في مواجهة التحديات، مقال

$$
\text { اقتصادي منشور علي التالي، www.rezgar.com. }
$$

(0) بوشول السعيد، واقع التكامل الاقتصادي لدول مجلس الخليج العربية وآفاقه، رسالة ماجستير،قسم الاقتصاد و علوم التسيير،كلية الحقوق والعلوم الاقتصادية، جامعة

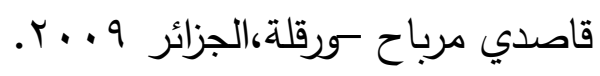

(T) جاسم بن محم القاسمي، آفاق التتمية والتكامل الاقتصادي بين دول الجزيرة العربية،

$$
\text { موسسة شباب الجامعة 1999، 199. }
$$

(V) بيلا بلاسا هو اقتصادي هنغاري، ولد في بوادبست في عام 9 ( I وتوفي في عام

19919، وعمل مع بول سامويلسون نظرية التكامل الاقتصادي (97 19 
(^) فؤاد أبوستيت، التكتلات الاقتصادية في عصر العولمة، القاهرة، الدار الدصرية

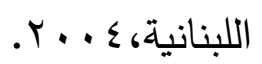

(9) محمد هشام خواجيكة، التكتلات الاقتصادية الدولية، جامعة حلب، مديرية

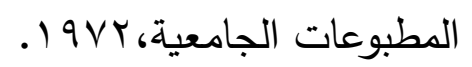
(10) Oxford :ClarendonPress,1922-1970. The Oxford Dictionary VII.C 